\title{
Retrieval of vertical wind profiles during monsoon from satellite observed winds over the Indian Ocean using complex EOF analysis
}

\author{
C M Kishtawal, Sujit Basu and S Karthikeyan \\ Meteorology and Oceanography Group, Space Applications Centre, Ahmedabad 380015, India.
}

\begin{abstract}
The aim of this paper is to study the feasibility of deriving vertical wind profiles from current satellite observations. With this aim, we carried out complex empirical orthogonal function (CEOF) analysis of a large number of radiosonde observations of wind profiles over the Indian Ocean during the monsoon months. It has been found that the first two CEOFs explain $67 \%$ of the total variance in wind fields. While the first principal component is well correlated with the winds at $850 \mathrm{mb}(r=0.80)$, the second one is highly correlated with winds at $200 \mathrm{mb}(r=0.89)$. This analysis formed the basis of a retrieval algorithm which ensures the retrieval of vertical profiles of winds using satellite tracked cloud motion vector winds. Under the assumption that accurate measurements of wind are available at the above mentioned levels, the r.m.s. error of retrieval of each component of wind is estimated to range between $2 \mathrm{~ms}^{-1}$ and $6 \mathrm{~ms}^{-1}$ at different levels, which is much less than the natural variance of winds at these levels. For a better visualization of retrieval, we have provided retrieved and true wind profiles side by side for four typical synoptic conditions during the monsoon season.
\end{abstract}

\section{Introduction}

Scale analysis suggests that the horizontal wind velocity is one of the most important parameters for numerical weather prediction (NWP) in the tropical regions (Haltiner and Williams 1980). It is well known that no direct relationship between mass and wind fields (such as geostrophic) exists over the tropics and the measurements of wind velocity are required to be made from independent observations. Unfortunately the distribution of surface based observing platforms e.g. land and coastal radiosonde stations, ships, buoys etc is far too insufficient to meet the requirements of NWP. In fact a significant fraction of oceans (particularly the Indian Ocean) still remains data-void and this situation is not likely to improve in the future. Space based observing platforms offer the only hope for providing the valuable wind information with desired coverage albeit with poor vertical resolution (only at two atmospheric levels at present using cloud tracking and at the ocean surface level using microwave scatterometer).

Remote sensing is the most direct approach to retrieve wind velocity from satellites over the data void oceanic regions. It is possible to obtain the wind velocity at lower and upper atmospheric levels (approximately $850 \mathrm{mb}$ and $200 \mathrm{mb}$ respectively) from satellite observations by tracking the movements of cloud tracers and these winds are termed as cloud motion vector (CMV) winds. There have been a number of attempts to develop and improve the generation, processing and utilization of CMV winds using geostationary satellite imageries. Development of operational systems like McIDAS (Man-Computer Interactive Data Analysis System, University of Wisconsin) and AOIPS (Atmospheric Oceanographic Information Processing System) has made it possible to retrieve a large number of cloud motion vectors, rapidly and accurately. Hubert and Whitney (1971) and Hubert and Timchalk (1972) showed that in the

Keywords. Complex EOF analysis; cloud motion vector winds; wind profiles; retrieval; monsoon. 
tropical regions synoptic scale low-level satellite derived CMV winds correspond closely to rawinsonde observations. Fujita et al (1975) showed the flow patterns of tropical circulation from CMV winds and Vieze et al (1972) found that the patterns of divergence and vorticity fields computed from CMV winds were consistent with the development, location and the movement of extratropical cyclone disturbances. Bauer (1976) and Suchman and Martin (1976) concluded that CMV winds could represent atmospheric motions within the accuracy of conventional data, for the synoptic scale. However, CMV winds include several sources of both systematic and random errors. Among them cloud top height determination is considered an important source of error. Many studies have estimated the errors of the satellite wind data sets over ocean and land areas showing that the errors lie within the range of accuracy of conventional wind data (Hubert and Timchalk 1972; Suchman and Martin 1976; Bauer 1976). Lee and Houghton (1984) showed quantitatively that the assignment of wind vectors to a single level introduced a local variability and systematic horizontal shears due to vertical wind shear effects, giving a variability comparable to that expected in natural mesoscale phenomena with $100 \mathrm{~km}$ length scale. With the advent of the first European remote sensing satellite ERS-1, the wind vectors are retrieved operationally at ocean surface using the microwave scatterometry. However these wind vectors have to be dealiased for the direction (at least for fast delivery wind products) and also wind speeds larger than $25 \mathrm{~ms}^{-1}$ cannot be verified using the active microwave instrument, at present. The ultimate goal for satellite wind studies is to identify how this information can be used to define the overall three-dimensional mesoscale wind fields. It is clear that the satellite derived winds are not sufficient by themselves for the NWP requirements, due to poor vertical resolution. In the present study we attempt to derive the vertical profiles of wind vector over the Indian Ocean during monsoon months using simulated satellite observations and the vertical structure functions of wind fields based on a large number of radiosonde observations.

Empirical Orthogonal Function (EOF) analysis creates by definition a set of structure functions which explain the inherent variability of the system under study with a minimum number of such functions (Lorenz 1959) and provides clues about the inherent physical processes involved in a system. The application of EOF analysis for earth sciences is well documented in the literature (Preisendorfer 1988). In a recent study, Kishtawal et al (1996) developed an algorithm for retrieving vertical wind profiles over the Indian Ocean using 2-level satellite winds. However in that study, the basic data used to develop the algorithm had only about $20 \%$ points during the monsoon seasons. Since the prediction of monsoon is of prime importance to the Indian subcontinent, the requirement of wind observations during the monsoon seasons is very crucial. In our present study, EOF analysis of a large data set of wind observations, containing only the monsoon winds of various years, have been performed in an attempt to understand the modes of variability of atmospheric wind fields during the monsoon over the Indian Ocean and to explore the possibility of retrieving vertical profiles of wind velocity using satellite observations.

Though the EOF analysis is well documented for the benefit of uninitiated readers we sketch below the salient features of the method. The aim of EOF analysis is to expand the parameter vector $V_{i}=V(t, r)$ (dependent on time $t$ and location $r$ of the measurement) in a series of empirical orthogonal vectors.

$$
\begin{aligned}
\mathbf{V}_{i}=\mathbf{V}(t, r)=\overline{\mathbf{V}} & +\sum_{k=1}^{N} C_{k, i} \mathbf{P}_{k}, \\
& i=1,2, \ldots \ldots \ldots \ldots \ldots M
\end{aligned}
$$

where $\overline{\mathbf{V}}$ denotes the horizontal and temporal mean of the parameter vector $\mathbf{V}_{i}, \mathbf{P}_{k}$ the vertical empirical orthogonal vector of order $k, M$ the total number of measurements and $N$ the degrees of freedom of the system. This approach of EOF analysis was successfully implemented by some researchers for single component geophysical parameter such as humidity (Wagner et al 1990; Basu et al 1995). In the present case our physical quantity of interest is horizontal wind, consisting of two components (zonal and meridional). To perform the EOF analysis of wind field we define a complex parameter $U$, termed as "pseudowind," so that

$$
U=u+i v
$$

where $u$ and $v$ are the zonal and the meridional components of wind respectively. Thus the analysis of the horizontal wind field may be carried out using complex empirical orthogonal functions (CEOFs).

The elements of the covariance matrix of the data set are:

$$
Z_{j k}=\overline{\left(U_{j}-\bar{U}_{j}\right)^{*}\left(U_{k}-\bar{U}_{k}\right)}
$$

where $j, k$ represent the index of level in the vertical direction, the asterisk denotes the complex conjugation and the overbar denotes the horizontal-temporal average. The principal information in $Z$ is contained in its low-order eigen structure. Since $Z$ is Hermitian by construction, it will possess real eigenvalues $\left(\lambda_{k}\right)$ and complex eigenvectors, $\mathbf{P}_{k}$. The corresponding EOF representation of $\mathbf{U}$ which optimally accounts for variance in pseudowind field is

$$
\begin{aligned}
U_{i}=\overline{\mathbf{U}}+\sum_{k=1}^{N} C_{k, i} \mathbf{P}_{k}, & \\
& \quad i=1,2, \ldots \ldots \ldots \ldots \ldots M
\end{aligned}
$$


where $N$ is the total number of eigenvectors and $C_{k i}$ are the (complex) principal components with $\overline{C_{m} C_{n}^{*}}=\lambda_{n} \delta_{m n}, \lambda_{n}$ being the eigenvalues and $\delta_{m n}$ being the Kroneker's delta. In other words, $\lambda_{n}=\overline{C_{n} C_{n}^{*}}$. After arranging the eigenvalues in descending order and normalizing the eigenvectors, the principal components for a specific anomaly vector $\mathbf{U}^{\prime}$ (departure from mean) can be computed in the following manner:

$$
C_{k}=\mathbf{P}_{k}^{\dagger} \mathbf{U}^{\prime}
$$

where the dagger denotes the Hermitian conjugation. The $n^{\text {th }}$ complex EOF has associated with it a fraction of the total field variance given by $\lambda_{n} / \Sigma \lambda_{n}$ (Preisendorfer 1988).

\section{Data and methodology}

Our analysis is based on a set of more than 1000 profiles of wind velocity belonging to the monsoon months (May, June, July and August) measured over the northern Indian Ocean (the Arabian Sea and the Bay of Bengal) as well as at coastal and island weather stations near the Indian peninsula for a period of six years (from 1984 to 1989). Besides that, the wind profiles measured during the Monsoon Experiment of 1979, MONEX-79 are also included in the data set. The geographical position of all observations is shown in figure 1 . The observations represent all the months of the monsoon seasons almost evenly, however in the spatial domain a majority of observations belong to coastal and island stations and about $40 \%$ of them are located over the open seas. The measured wind profile contains wind velocity at every $50 \mathrm{mb}$ between $1000 \mathrm{mb}$ and $200 \mathrm{mb}$ (17 levels). However in our data

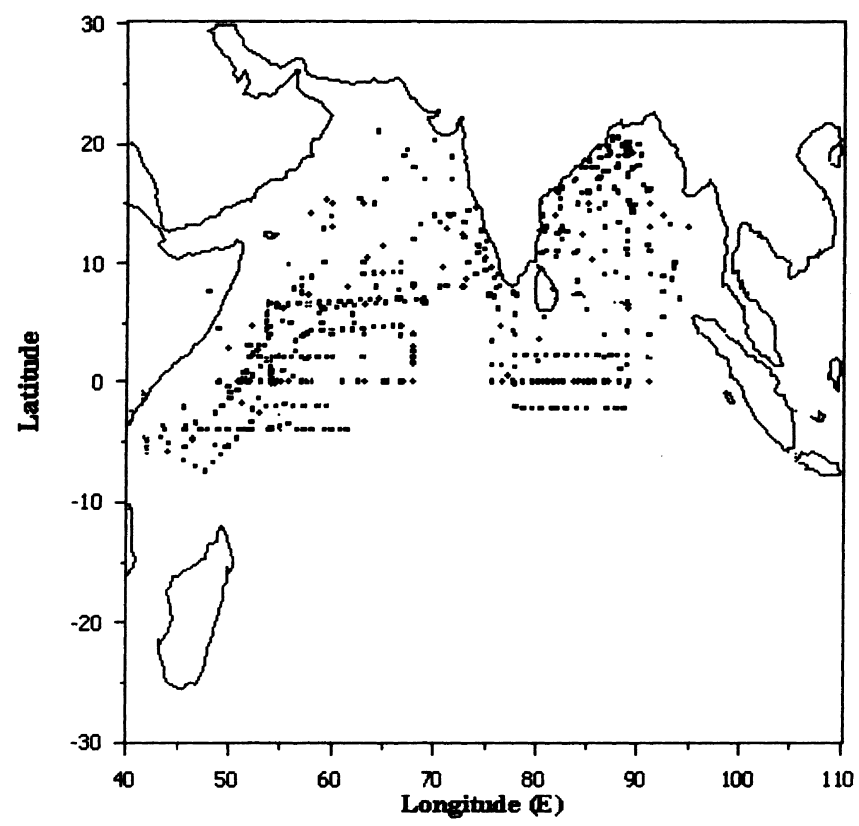

Figure 1. Locations of ship and coastal/island stations. set we occasionally found the data missing at different levels at different times. Only those profiles are used in the analysis for which the observations are available at least at 15 levels out of 17 , with a condition that the observations should not be missing at two consecutive levels. The data gaps are removed using simple linear interpolation, separately for each component of wind.

According to (2) the original parameter vector $\mathbf{U}_{i}$ can be reconstructed by means of a linear combination of complex eigenvectors $\mathbf{P}_{k}$ weighted by the principal components $\mathrm{C}_{k i}$ (and of course after adding the mean vector). If (2a) is truncated after $n$ terms, we get an approximation $\mathbf{U}_{i n}$ of the original measurements $\mathbf{U}_{i}$. The mean error $F_{n}$ of this approximation is given by

$$
F_{n}=\left[\frac{1}{M} \sum \mathbf{f}_{i, n} \mathbf{f}_{i, n}\right]^{1 / 2}
$$

where $\mathbf{f}_{i, n}$ is defined as

$$
\mathbf{f}_{i, n}=\mathbf{U}_{i}-\mathbf{U}_{i, n}=\sum_{k=n+1}^{N} C_{k, i} \mathbf{P}_{k} .
$$

For computing complex EOFs, the mean profile of the complex parameter $\mathbf{U}$ is subtracted from the individual measured profiles of $U$, the covariance matrix is constructed according to (1) and the corresponding eigenvalue problem is solved numerically.

The results of the complex EOF analysis are shown in figure 2. The first eigenvector explains $45 \%$ of the

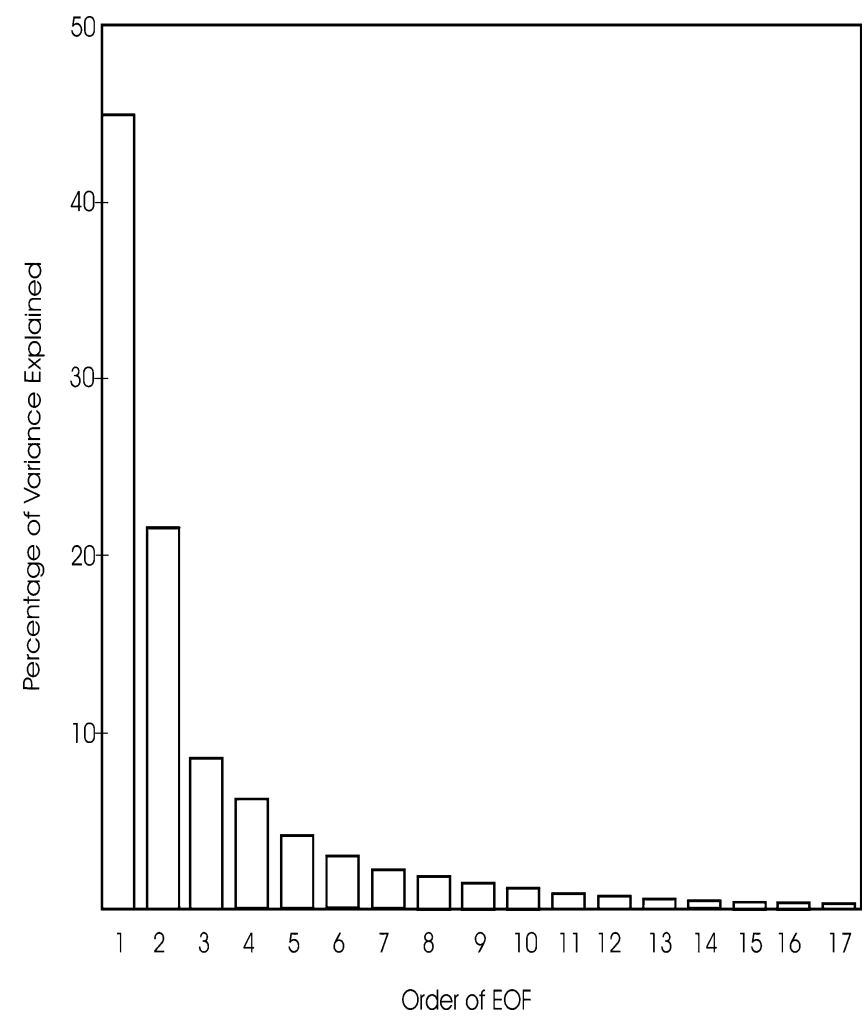

Figure 2. Percentage of variance explained by different CEOFs. 
(a)

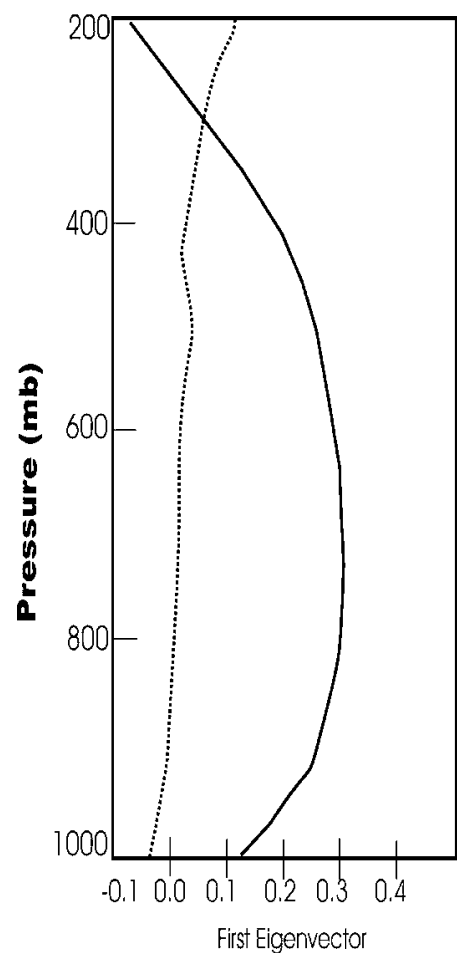

(b)

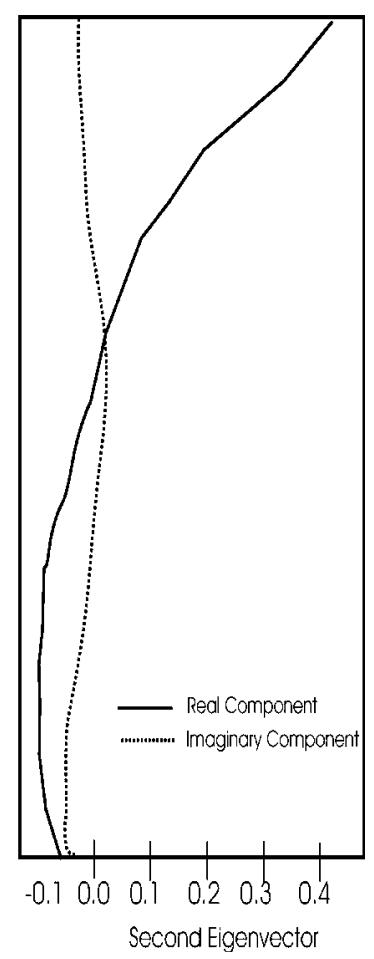

Figure 3. Vertical profiles of first two eigenvectors (solid line - real component; dashed line -' imaginary component).

total variance over the Indian Ocean, as shown by the normalized eigenvalues, while the second eigenvector explains about $22 \%$, so the first two eigenvectors together explain more than $67 \%$ of the total variability in wind fields. The eigenvector of third order explains less than $10 \%$ of the total variability and it seems justified to utilize only the first two dominant eigenvectors for approximating a wind profile reasonably. Figure 3 ( $\mathrm{a}$ and $\mathrm{b}$ ) shows the vertical distribution of real and imaginary parts of the first two (most dominant) eigenvectors. It seems that the real parts of these two eigenvectors are almost an order of magnitude larger than the corresponding imaginary parts. We can attempt to explain the modes of wind variability based on the vertical structure of real parts of these EOFs. It shows that between $1000 \mathrm{mb}$ and $300 \mathrm{mb}$, the dominant variability of the wind profile is governed by a positive correlation between the layers. Above $300 \mathrm{mb}$ winds show a negative correlation with winds below $300 \mathrm{mb}$. The EOF of first order has a positive maximum at $800 \mathrm{mb}$, and a minimum (negative maximum) at $200 \mathrm{mb}$ which shows a tendency to extend to upper levels. These may be identified with the average position of westerly and easterly flows at upper and lower troposphere respectively, over the Indian Ocean in monsoon regime. The second eigenvector shows a weak negative correlation between the winds below $600 \mathrm{mb}$ and those above $600 \mathrm{mb}$, while the winds above $600 \mathrm{mb}$ are strongly correlated. A gradual reduction of the first eigenvector between

$850 \mathrm{mb}$ and the surface may be attributed to the frictional influence of the planetary boundary layer.

Stability is a necessary condition for the intended use of the EOF-analysis in atmospheric sciences. Due to the finite number of measurements, the deduced CEOFs are not necessarily representative of all possible occurring synoptic situations. Eigenvectors are termed unstable if their structure changes in case the set of measurement vectors is increased or reduced. A measure for the stability of the complex eigenvector $\mathbf{P}_{k}$ is given by the ratio of the sampling error $\delta \lambda_{k}$ of the eigenvalue $\lambda_{k}$ to the differences between two successive eigenvalues $\left(\Delta \lambda_{k}=\lambda_{k}-\lambda_{k+1}\right)$. Thus sampling error $\delta \mathbf{P}_{k}$ of the eigenvector $\mathbf{P}_{k}$ is defined as (North et al 1982):

$$
\delta \mathbf{P}_{k}=\frac{\delta \lambda_{k}}{\Delta \lambda_{k}} \mathbf{P}_{k+1}
$$

With $\delta \lambda_{k}=\lambda_{k}(2 / M)^{1 / 2}, M$ is the total number of independent measurements. An eigenvector is termed stable if the ratio $\delta \lambda_{k} / \Delta \lambda_{k}$ is significantly smaller than one. In our case this ratio for the first two complex eigenvectors is 0.091 and 0.073 respectively, which shows that the corresponding eigenvectors are stable.

In order to underline the physical relevance of the dominant eigenvectors, we investigate the relationship between the principal components and winds at various levels. Now since both the principal component and the pseudo-wind $(\mathbf{U})$ are complex, the correlation coefficient between the pseudowind at a particular level and the principal component of a particular order is:

$$
\begin{aligned}
& |r|_{l}= \\
& \frac{\left|1 / M \sum_{i}\left(U_{i, l}-\bar{U}\right)\left(C_{i}-\bar{C}\right)^{*}\right|}{\left[1 / M \sum_{i}\left|\left(U_{i, l}-\bar{U}\right)\right|^{2}\right]^{1 / 2}\left[1 / M \sum_{i}\left|\left(C_{i}-\bar{C}\right)\right|^{2}\right]^{1 / 2}}
\end{aligned}
$$

where $U_{i, l}$ signifies the pseudowind at $l$ th level and at horizontal-temporal location $i$ while $C_{i}$ is the principal component of a particular order (1st or 2nd) corresponding to the same location.

Figure 4 shows the correlation coefficients of first and second principal components with the pseudowinds at various levels. The first order coefficient $C_{1}$ is highly correlated with the pseudo-winds at levels between $850 \mathrm{mb}$ and $600 \mathrm{mb}(r=0.8)$ and this correlation decreases rapidly for levels above $400 \mathrm{mb}$. The second principal component $C_{2}$ shows a high correlation with pseudowinds at $200 \mathrm{mb}(r=0.9)$.

At this point of discussion it would be worthwhile to give a brief description of the average wind fields over the Indian Ocean. Before the month of April the low level winds $(850 \mathrm{mb})$ follow an easterly trade wind pattern while upper level winds $(200 \mathrm{mb})$ are mostly westerly in the northern hemisphere. The wind 


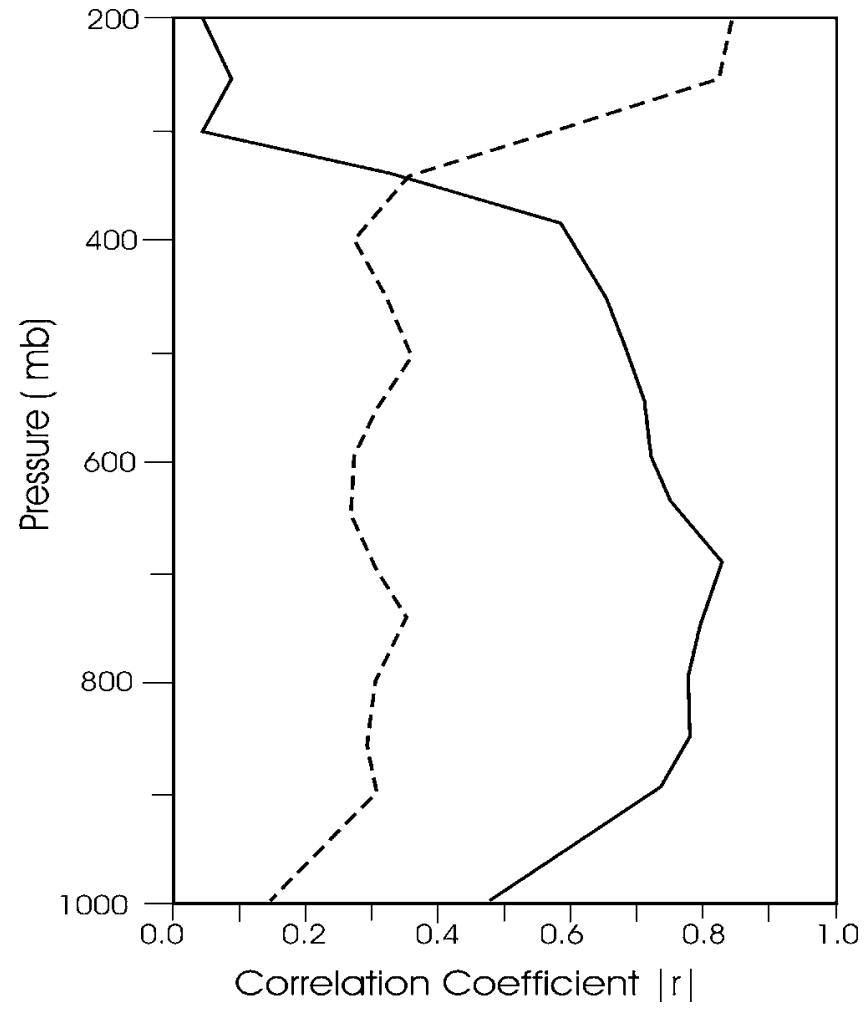

Figure 4. Correlation of principal components (PC) of first and second orders with pseudowinds at different vertical levels (solid line: PC-1, dashed line: PC-2) patterns at both levels start showing the tendency for reversal by the month of April due to the combined effects of the northward transition of the Inter Tropical Convergence Zone (ITCZ) and the Coriolis effect (Ramage and Raman 1972; Prasad et al 1991). By the month of May, the wind flow at low levels becomes westerly which is curved around the monsoon trough, located along the east coast of India. At upper levels, the wind patterns are predominantly easterly in the Northern Hemispheric Indian Ocean. The flow patterns at both the levels become streamlined and intense by the month of June when the monsoon gets established over the Indian landmass and is followed by heavy precipitation. A low level westerly jet begins to develop at the core of monsoon winds by the month of June along $15^{\circ}$ latitude line and acquires full strength with peak winds reaching up to $25 \mathrm{~m} / \mathrm{s}$ in the month of July. An easterly jet also develops at the upper levels by the month of June approximately parallel to $5^{\circ}$ latitude and gains full strength of about $40 \mathrm{~m} / \mathrm{s}$ in the month of July. After the withdrawal of the monsoon in the month of September, the wind patterns slowly revert back to the pre-April situation.

A latitude-pressure cross section at $75^{\circ} \mathrm{E}$ shows the main features of the zonal and meridional circulation at the monsoon region for January and July (figure 5 $\mathrm{a}, \mathrm{b}, \mathrm{c}, \mathrm{d})$. Near the equator, the zonal circulation is

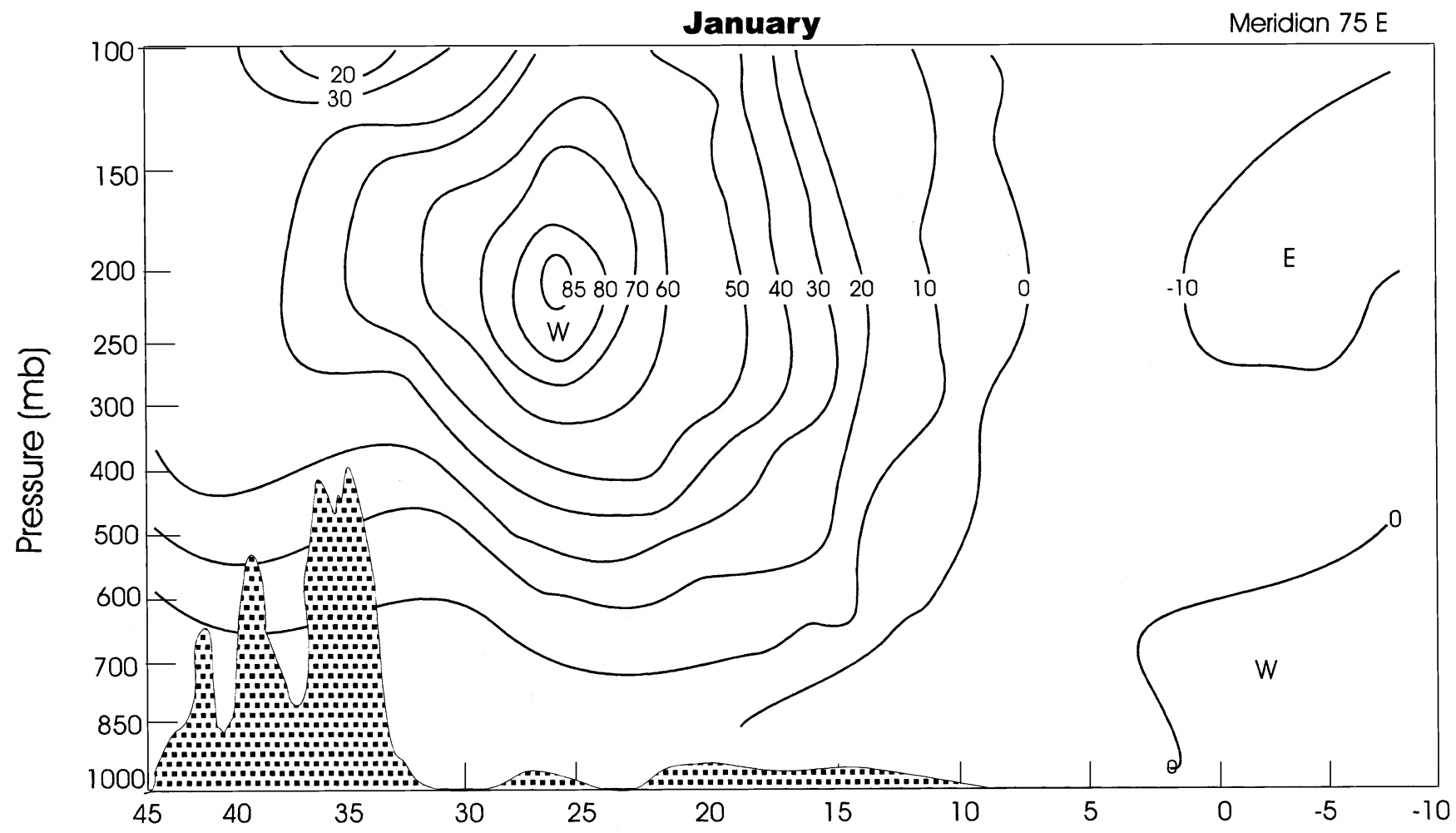

(a)

Latitude (N)

Figure 5. (Continued). 


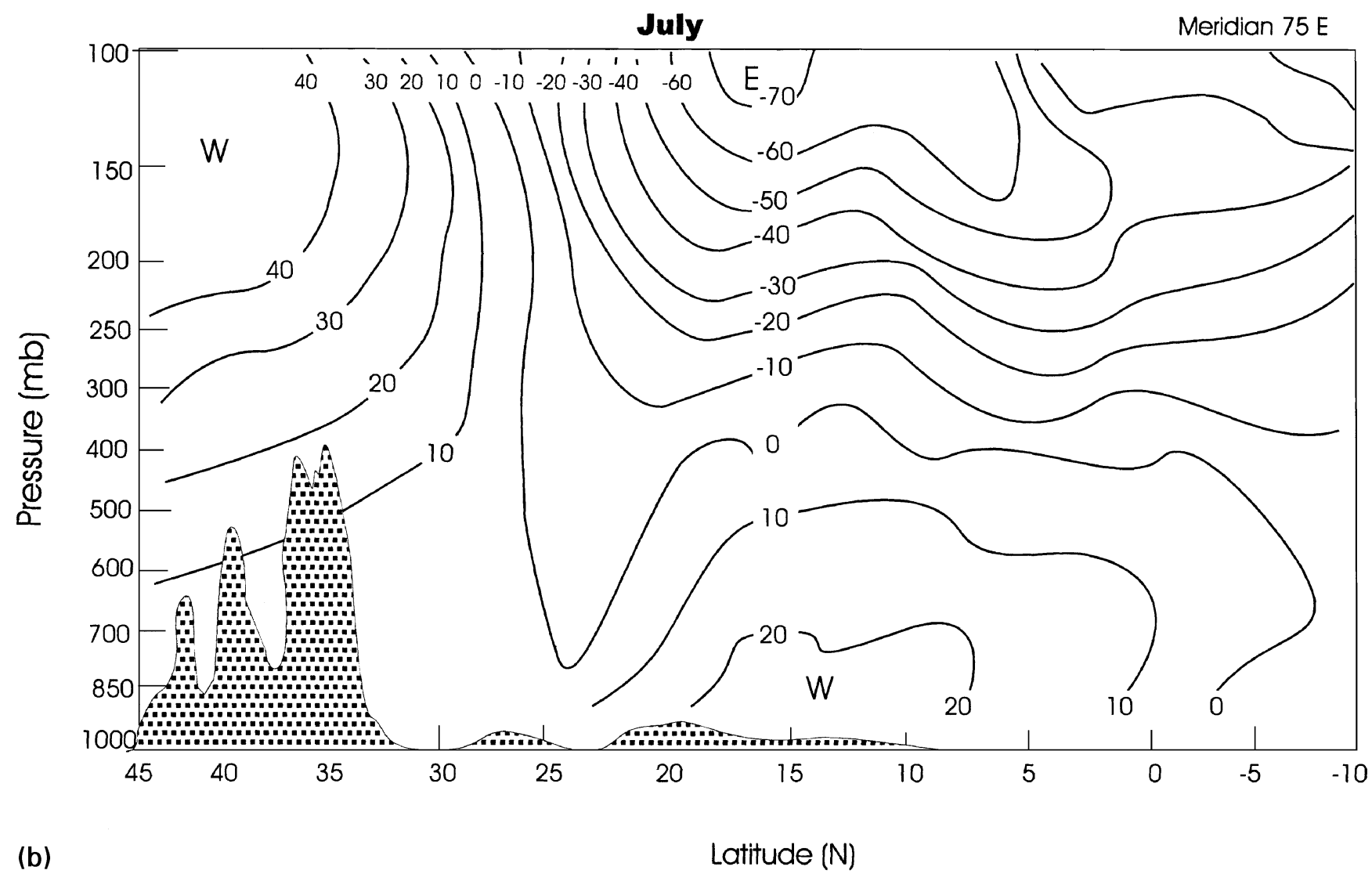

January

Meridian $75 \mathrm{E}$

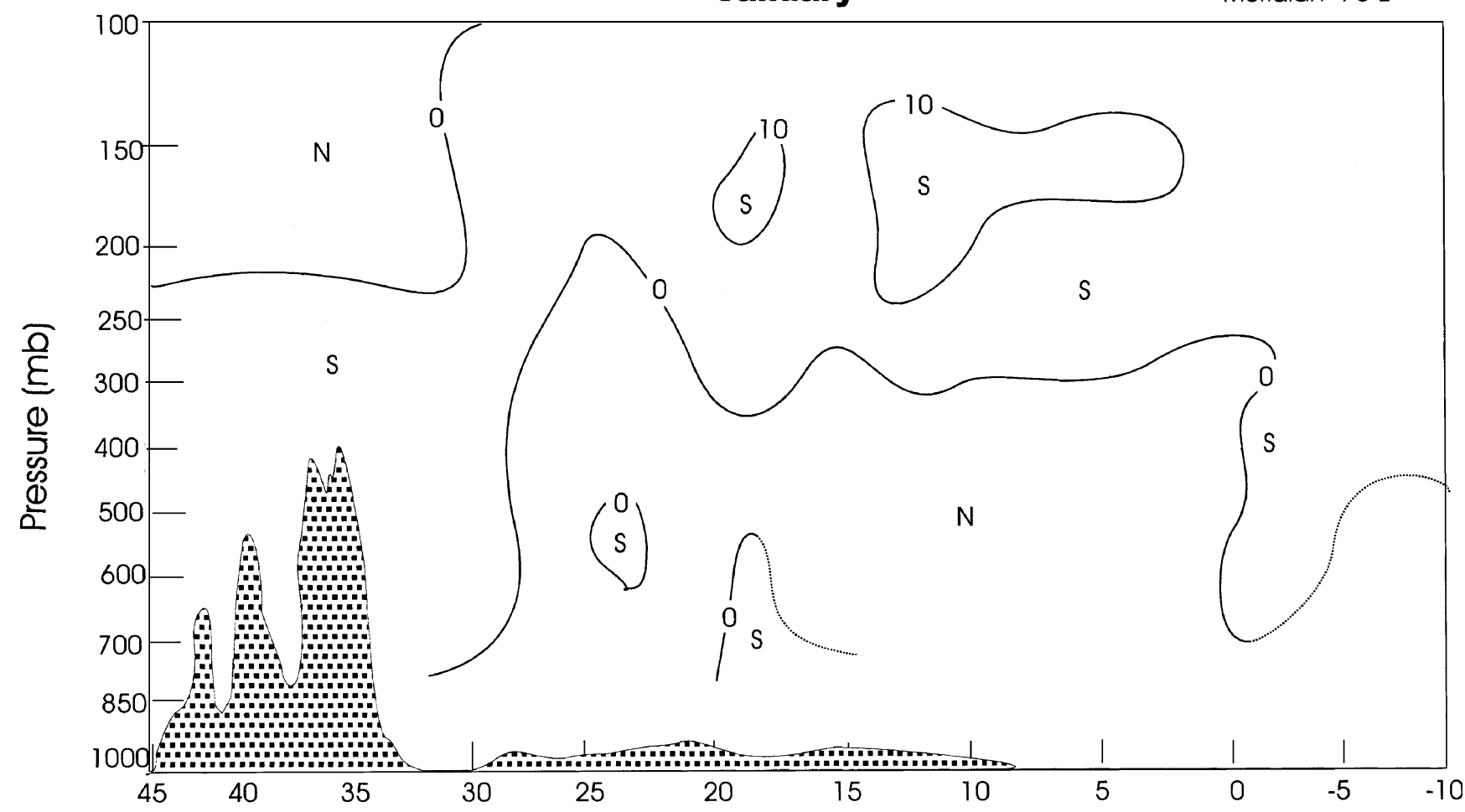

(c)

Latitude (N)

Figure 5. (Continued) 


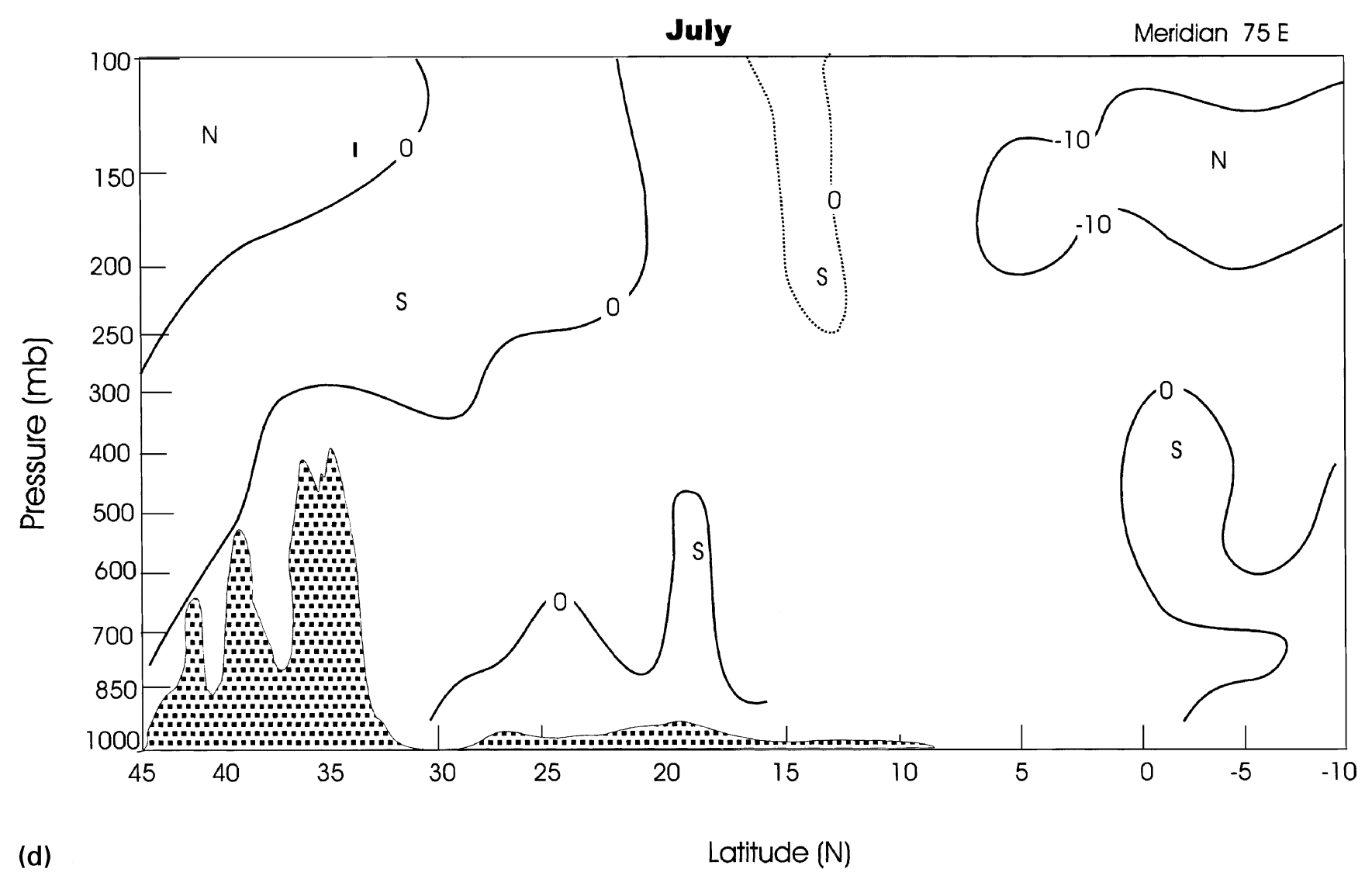

Figure 5(a-d). (a) Latitude-pressure cross section of zonal wind at $75^{\circ}$ E longitude for the month of January; (b) same as in figure 5(a) but for the month of July; (c) same as figure 5(a) but for the meridional wind; (d) same as figure 5(b) but for the meridional wind. (Figure reproduced from Ananthkrishnan 1975).

westerly in the lower troposphere and easterly aloft throughout the year. The depth of the lower tropospheric westerlies increases during the summer monsoon months. The upper easterlies attain the maximum strength in this season, speeds exceeding $25 \mathrm{~ms}^{-1}$ near $150 \mathrm{mb}$ level which is a little below the tropopause. North of $10^{\circ} \mathrm{N}$, the westerly winter circulation begins to predominate and the duration of the easterly circulation decreases. At higher latitudes, near $30^{\circ} \mathrm{N}$, the tropospheric circulation is practically confined to the months of July and August. Further north, the westerly circulation prevails throughout the year.

During the summer monsoon, the meridional circulation is directed from north to south in upper troposphere, and at the south of $25^{\circ} \mathrm{N}$, while at upper latitudes of India it is from south to north (Ananthakrishnan 1975). The summer meridional flow from southern to northern hemisphere is very strong in the upper troposphere over Gan Island. Zonally averaged meridional and vertical wind velocities show that the winter Hadley cell of the southern hemisphere has its ascending branch displaced into the northern hemisphere during northern summer. The displacement reaches a maximum over south Asia in confor- mity with the large northward shift of the ITCZ over this region in summer.

EOF analysis of pseudo-wind fields over the Indian Ocean suggests two important mechanisms. Since the first principal component is highly correlated with the pseudowind at $850 \mathrm{mb}$, arguably wind at this level accounts for the highest degree of variability. Similarly a high correlation of second principal component with low level winds (200 mb) suggests that the atmospheric circulation in the Indian Ocean is dominated by two primary modes controlled by some physical processes taking place at these two levels.

\section{Profile retrieval algorithm}

Incidentally the coefficients $C_{1}$ and $C_{2}$ show maximum correlation with the winds at the levels where it is usually possible to make wind measurements through satellite observations. CMV winds are derived from the imageries of geostationary satellites at upper (about $200 \mathrm{mb}$ ) and lower (about $850 \mathrm{mb}$ ) atmospheric levels, although the assignment of pressure levels to the derived winds is not always exact. If it is possible to derive information about 
$800 \mathrm{mb}$ and $250 \mathrm{mb}$ winds from satellite observations, it may be concluded from the preceding discussion that these observations should lead to a determination of expansion coefficients and to an estimation of wind profile according to a truncated EOF expansion. This is the basic idea underlying the retrieval algorithm. The quantities $U_{850}$ (pseudowind at $850 \mathrm{mb}$ ) and $U_{200}$ can now be written in a restricted EOF expansion form as

$$
\left[\begin{array}{l}
U_{850}-\bar{U}_{850} \\
U_{200}-\bar{U}_{200}
\end{array}\right]=\left[\begin{array}{ll}
P_{1,850} & P_{2,850} \\
P_{1,200} & P_{2,200}
\end{array}\right]\left[\begin{array}{l}
C_{1} \\
C_{2}
\end{array}\right]
$$

where $P_{1,850}$ is the component of the first eigenvector belonging to the $850 \mathrm{mb}$ level and so on, and the quantities with overbars represent horizontaltemporal averages. The parameters of the column vector on the left hand side are available from satellite measurements while those of the matrix on the right hand side can be known from the EOF analysis of a large data set of "true" wind profiles converted to pseudowinds. The solution of matrix equation (7) leads to determination of $C_{1}$ and $C_{2}$. A second order approximation of pseudo-wind profiles then follows according to the EOF expansion as:

$$
\mathbf{U}_{2}=\overline{\mathbf{U}}+\sum_{k=1}^{2} C_{k} \mathbf{P}_{k}
$$

where $\mathbf{U}_{2}$ is the pseudowind profile of the second order approximation. It should be noted that the coefficients $C_{k}$ are close but not identical to the principal components derived from EOF analysis. Furthermore, the accuracy of retrieved profiles depend on the accuracy of input observations.

We would like to point out here the difference between our approach and the regression technique. Wind at each level could alternately have been retrieved via standard linear regression using the two independent observations $U_{200}$ and $U_{850}$, however, it is quite obvious that the winds at different levels obtained by regression approach will not be mutually consistent because the statistical correlations existing between winds at different levels are not taken explicitly into account in such an approach.

In contrast, the EOF expansion, even a truncated one, explicitly takes into account the statistical correlation (at least a very significant part of it) existing between winds at different levels. After all the EOFs are nothing but the eigenvectors of covariance matrix. Overall, we conclude that a single wind profile derived by the EOF approach is much more suitable for inclusion in NWP models than wind values obtained by a number of regressions which are not necessarily related to each other in a statistically significant manner. As regards the use of EOF based retrieval we would like to further add that such an approach has already been utilized by other researchers for retrieving humidity profiles from satellite

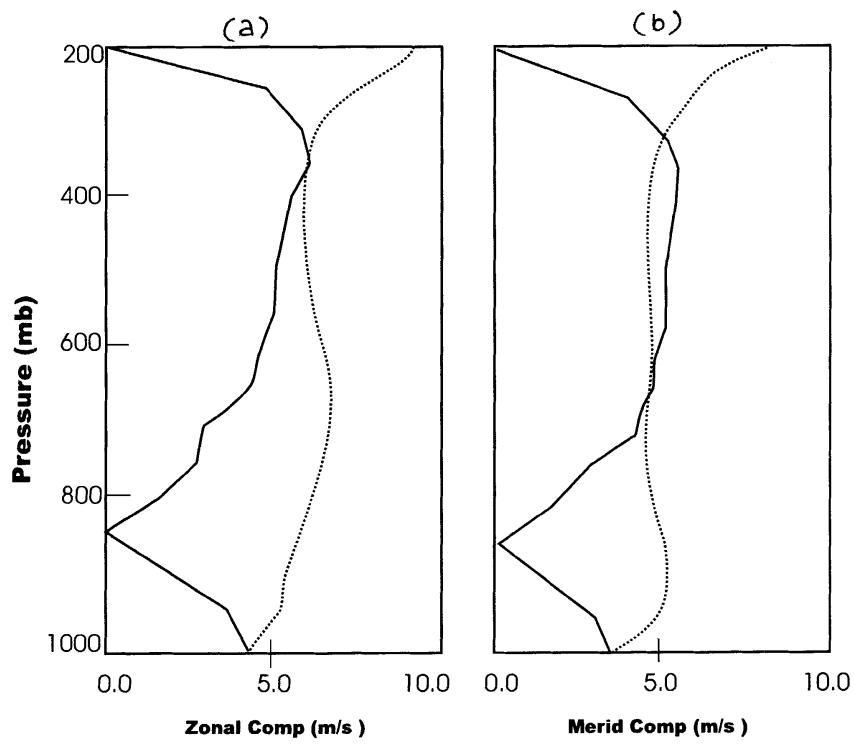

Figure 6. RMS errors of retrieved winds (solid line) and their comparison with the natural variance of wind (dashed line) (a) zonal component; (b) meridional component.

derived total precipitable water (Wagner et al 1990; Basu et al 1995).

The retrieval algorithm was tested using a test set of about 200 wind profiles not used for determining the set of EOFs. Using relationship (8) we have derived the vertical profiles of pseudowinds and then the zonal and the meridional components of wind were separated as these belong to the real and the imaginary parts of pseudowind. It was assumed that the observations of wind at 850 and $200 \mathrm{mb}$ are available exactly. The retrieved profiles were then compared with actually observed profiles and the root mean square errors of retrieval were computed. Figure (6a) shows the r.m.s. errors of retrieval at each level. It shows that the error of estimation is less than $5 \mathrm{~m} / \mathrm{s}$ for meridional component of wind as well as for the zonal component of the wind at most of the levels. Near 850 and $200 \mathrm{mb}$ levels (where the exact observations are supposed to be available) the errors of estimation are substantially lower. The maximum r.m.s. error of estimate for meridional wind component is $5.4 \mathrm{~m} / \mathrm{s}$ which occurs near $400 \mathrm{mb}$ while the maximum error of $5 \mathrm{~m} / \mathrm{s}$ in zonal component of wind occurs near $400 \mathrm{mb}$ and the surface. A comparison of r.m.s. errors of estimate with the standard deviation of data set shows that the skill of the retrieval algorithm is very good for the zonal component of the wind where the r.m.s. error of retrieval is significantly below the natural variance of the wind. In case of meridional wind component, we have poor retrieval skill in the atmospheric layer between $500 \mathrm{mb}$ and $350 \mathrm{mb}$, whereas, at other levels the skill is reasonably good. At $1000 \mathrm{mb}$, or near the surface, the r.m.s. error of retrieval for both the components of wind is as large as their natural variance. However, the surface level 
(A)

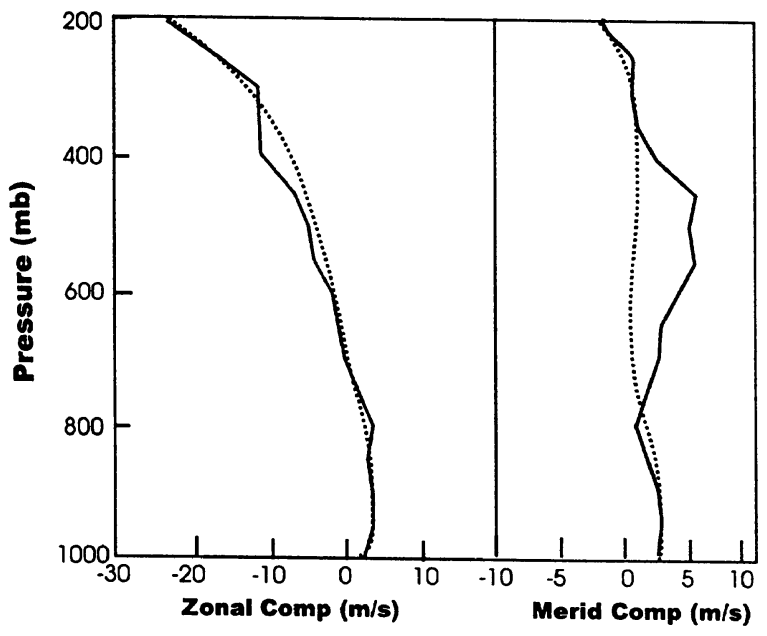

(C)

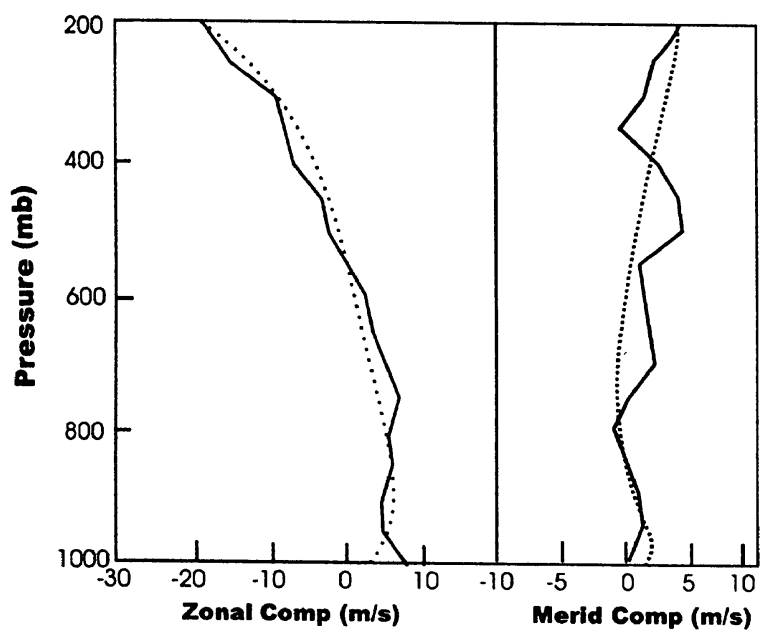

(B)

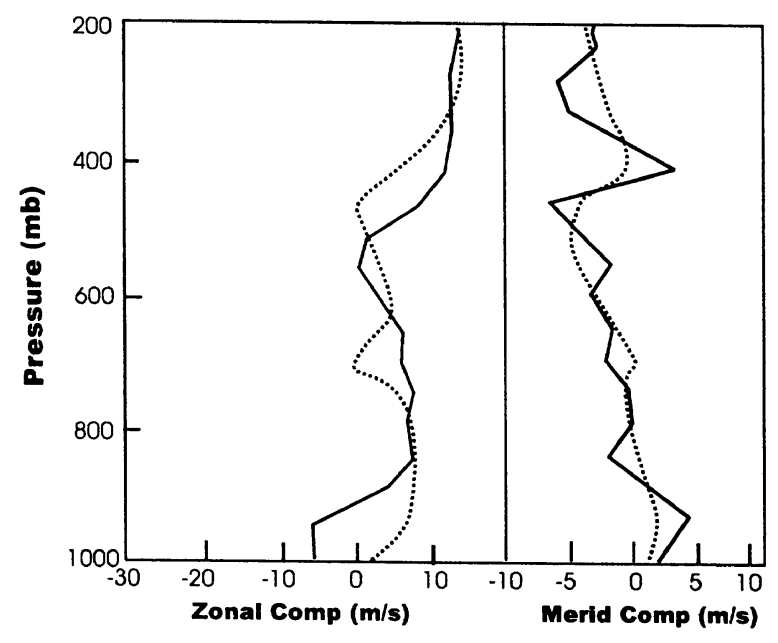

(D)

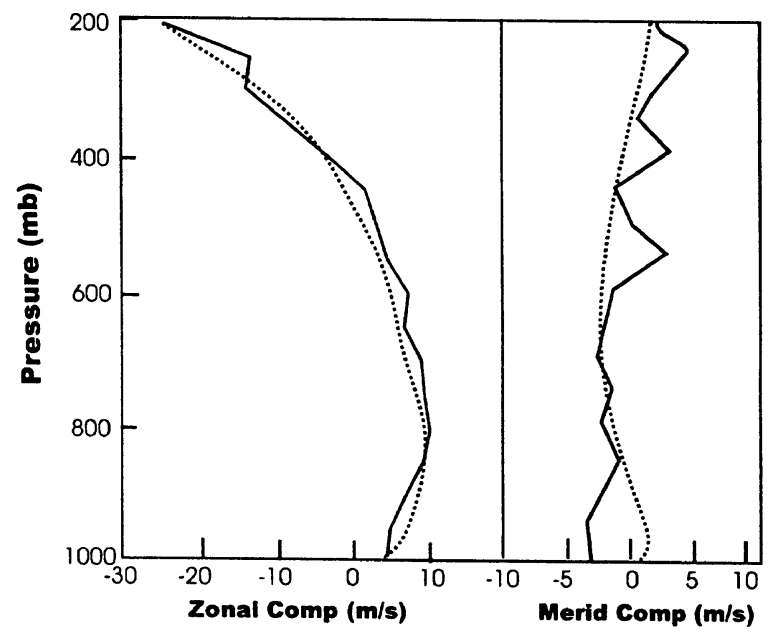

Figure 7. Comparison of retrieved and actual wind profiles. Left part of each figure represents zonal component while the right part represents meridional component of wind (solid line: zonal component, dashed line: meridional component of wind).

measurements of wind velocity are now available on operational basis from the observations of microwave scatterometers, so the loss of skill in retrieving these winds using CMVs is not a serious problem from the operational point of view.

However, the errors of retrieval can be expected to be somewhat more than those expressed in previous paragraph. This is due to the fact that the actual CMV winds themselves have some inherent errors of retrieval. Woik et al (1992) concluded that there is a bias of $\sim 2.5 \mathrm{~ms}^{-1}$ between radiosonde and CMV winds, while Lunnon and Lowe (1992) showed that the mean difference between CMV and operational analysis was $\sim 3 \mathrm{~ms}^{-1}$. These errors may affect the accuracy of retrieved wind profiles using the EOF method. However, these errors can be modeled by the EOF algorithm itself, if one uses a combined data set of CMV and replacing CMV winds at 200 and $850 \mathrm{mb}$ levels, and keeping the radiosonde winds at other levels to form a large hybrid data set, for EOF computation. This hybrid approach is expected to take care of bias and to some extent other systematic differences between CMV and radiosonde winds.

Figure 7 (A to D) show comparisons of retrieved wind profiles with the actually observed profiles for different synoptic conditions using only the first two EOFs. Both the zonal and the meridional components are shown. These profiles represent typical synoptic situations over the Indian Ocean during the monsoon. Figure 7(b) shows a situation where a shear flow is developing near $500 \mathrm{mb}$. Figure $7(\mathrm{~d})$ shows a well developed upper level easterly flow with wind speeds close to $30 \mathrm{~ms}^{-1}$, while at lower levels the flow is weak. For all these cases, both the zonal and the meridional components of the retrieved wind profiles show a reasonably good agreement with the "true" wind profiles. The fit of zonal component is much better than that of the meridional component. This is due to 
the fact that the variance of meridional wind component with respect to its mean value is much larger than that for the zonal wind during the monsoon season. The retrieved profiles show a smooth vertical trend as compared to the observed profile, mainly because of the low order truncation of our algorithm.

Presently various operational weather prediction centres use the analytical vertical structure functions (e.g., eq. (53) of Barewell and Bromley 1988) to produce the analysis of winds at different vertical levels using CMV winds. However, such functions can not be guaranteed to be consistent with the statistical behavior of wind profiles at different geographical locations. We believe that if EOFs based on a large data set were used instead of generalized empirical functions during the objective analysis procedure in a data assimilation cycle, it will be possible to produce wind profiles with higher statistical consistency. In fact, similar analytical structure functions were earlier proposed for deriving vertical humidity profile also. However, because of the inadequacy of this approach, researchers have become inclined to the use of EOFs (Wagner et al 1990; Basu et al 1995; Kishtawal et al 1996). Moreover, with the availability of more radiosonde data and with increasingly strict quality control of such data, even the EOFs may be updated in future.

In the future, wind profile retrieval might be possible using Lidar Doppler atmospheric wind sounders. However, it is still not quite clear whether such a sensor will be able to provide wind at all important atmospheric pressure levels. Our study shows that even if a sensor were able to provide the wind measurements at two levels where we have obtained maximum correlation between principal components and pseudowinds, it might be feasible to determine the vertical wind profiles with reasonable accuracy.

\section{References}

Ananthakrishnan R 1975 Vertical structure of the atmosphere over India and adjoining areas Geophysical Fluid Dynamics Workshop on Topics in Monsoon Meteorology; Indian Institute of Sciences, Bangalore, July, 1975.

Barewell B R and Bromley R A 1988 The adjustment of NWP models to the local perturbations; $Q J R$ Met Soc 114 665-689

Basu S, Gairola R M, Kishtawal C M and Pandey P C 1995 EOF analysis of humidity profiles over the Indian
Ocean and an assessment of their retrievability using satellite microwave radiometry; $J$ Geophys Res $\mathbf{1 0 0}$ 23009-23017

Bauer K G 1976 A comparison of cloud motion winds with coinciding radiosonde winds; Mon. Weather Rev. 104 407413

Fujita T T, Pearl E W and Shenk S E, 1975 Satellite tracked cumulus velocities; J Appl Meteorol 14 407-413

Haltiner G J and Williams R T 1980 Numerical weather prediction and dynamic meteorology (New York: John Wiley Publ.) 477 pp.

Hubert L F and Whitney L F Jr 1971 Wind estimations from geostationary satellite pictures; Mon. Weather Rev. 99 $665-672$

Hubert L F and Timchalk A 1972 Convective clouds as tracers of air motion; NOAA Tech. Memo. NESS-40, 22 pp.

Kishtawal C M, Basu S and Pandey P C 1996 An algorithm for retrieval of vertical wind profiles from satellite observed winds over the Indian Ocean using complex EOF analysis; $J$. Appl. Meteorol. 35 532-540

Lee D K and Houghton D D 1984 A quantitative study of satellite winds for mesoscale meteorology; Mon. Weather Rev. 112 990-1004

Lorenz E M 1959 Empirical orthogonal functions and statistical weather prediction. Final rep. Statistical Forecasting Project, 1959; Massachusetts Institute of Technology, Dept. of Meteorology, 29-78

Lunnon R W and Lowe DA 1992 Spatial Scale dependency of errors in satellite cloud tracked winds; Adv. Space. Res. 12 $127-131$

North G R, Bell T L, Chalan R F 1982 Sampling errors in the estimation of empirical orthogonal functions; Mon. Weather Rev. 110 699-706

Pelson, C A 1980 Short interval SMS wind vector determinations for a severe local storm area; Mon. Weather Rev. 108 1407-1418

Prasad S, Khanna P N, Rao, A V K R and Kelkar R P 1991 Satellite derived monthly average wind fields over the Indian Ocean in April-July 1988; Mausam 41 445-450

Preisendorfer R W 1988 Principal component analysis in Meteorology and Oceanography. Developments in Atmospheric Sciences, Vol. 17, Elsevier Science, (eds) V Mobley and Curtis 425 pp.

Ramage C S and Raman C R V 1972 Meteorological atlas of the Indian Ocean Expedition. Vol. 2 (Upper air) National Science Foundation, USA.

Suchman D and Martin D, 1976 Wind sets from SMS images: An assessment for quality of GATE; Journal of Applied Meteorology 15 1265-1278

Vieze W, Serebreney S M and Mancuso R L 1972 A sample computation of kinematic properties from cloud motion vectors; Journal of Applied Meteorology 11 731-741

Wagner D, Ruprent E and Simmer C 1990 A combination of microwave observations from satellites and an EOF analysis to retrieve vertical humidity profiles over the oceans; Journal of Applied Meteorology 29 1142-1157

Woik H 1992: Verification of cloud motion winds at global scale; Adv. Space Res. 12 115-118 